\title{
A Randomized Clinical Trial of a Computer Based Preventive Intervention: Replication and Extension of ePREP
}

\author{
Scott R. Braithwaite and Frank D. Fincham \\ Florida State University
}

\begin{abstract}
This randomized clinical trial replicated the efficacy of the ePREP preventive intervention for mental health and relationship relevant outcomes in a sample of 77 college students. It extended previous research by demonstrating efficacy at a 10-month follow up. Participants in the ePREP condition experienced improved mental health and relationship relevant outcomes relative to those who received a placebo intervention. The impact of the ePREP intervention on these outcomes was durable to relationship dissolution with and without repartnering. The flexibility of this intervention empowers it to overcome key obstacles in the dissemination of relationship education.
\end{abstract}

Keywords: computer-based interventions, prevention, relationship education

A handful of relationship education programs have demonstrated efficacy in reducing marital discord and divorce (Jakubowski, Milne, Brunner, \& Miller, 2004). However, two key problems face prevention focused relationship education. First, research indicates that those who usually receive relationship education are at relatively low risk for divorce and other marital problems (Sullivan \& Bradbury, 1997). Second, longitudinal studies that have examined the impact of relationship education over longer spans of time suggest that the gains achieved through relationship education tend to diminish over time (Markman, Renick, Floyd, $\&$ Stanley, 1993). Consequently, a need has arisen to produce forms of evidence based relationship education that address these two limitations by increasing flexibility without sacrificing efficacy.

At least three interventions have arisen to meet this need. Halford and colleagues' Couple CARE program (Halford, Moore, Wilson, Dyer, \& Farrugia, 2004), which allows participants to complete most of the work associated with the intervention on their own in combination with telephone sessions with a therapist, demonstrated improved relationship satisfaction relative to controls. Larson and colleagues examined the impact of an assessment based premarital intervention (RELATE) and found that self-guided use of this intervention produced increased relationship satisfaction relative to controls (Larson, Galbraith, Holman, \& Stahmann, 2007). Research on both of these interventions is limited by the fact that these studies provided only short-

Scott R. Braithwaite, Department of Psychology and Family Institute, Florida State University; Frank D. Fincham, Family Institute, Florida State University.

Correspondence concerning this article should be addressed to Scott R. Braithwaite, Department of Psychology, Florida State University, Tallahassee, FL 32306-4301. E-mail: srbraithwaite@ gmail.com term pre and post intervention data, and neither examined the impact of these interventions on other relevant variables such as psychopathology.

One potentially powerful means of dissemination is to offer programs via computers and computer-based networks. Importantly, a meta-analysis conducted by Cavanaugh and Shapiro (2004) found that clients who have received computer-based interventions see them as a valid form of treatment. Brief, individual focused relationship interventions have the power to overcome many of the obstacles that currently face dissemination of relationship education. These interventions could benefit the relationships of individuals who have partners that are averse to receiving couples treatment as usual. Moreover, the potential cost effectiveness and flexibility offered by computerbased treatments allow them to reach nearly any population and provide an attractive alternative for important populations that relationship educators would like to reach, including technology savvy adolescents and those who have negative attitudes about face-to-face relationship education or treatment.

ePREP, a computer based intervention developed by Braithwaite and Fincham (2007), is designed to be used as a primary preventive intervention and a flexible form of relationship education. In an initial trial, ePREP showed promise in improving key outcomes such as problematic communication, intimate partner violence, depression, and anxiety in a randomized control trial that followed participants for approximately 2 months. In this study, ePREP participants demonstrated better mental health and relationship outcomes than the placebo/control condition. Moreover, the mental health outcomes for those in the ePREP condition were not significantly different from another condition that used a computerized empirically supported preventive intervention designed specifically to address depression and anxiety (Cukrowicz \& Joiner, 2007). Critical to establishing the viability of ePREP, however, is replication 
of the initial findings. Further, additional data are needed to determine the longer-term consequences of this intervention and how they may be moderated by relationship dissolution. Thus, the present study seeks to replicate the impact of ePREP and to examine its efficacy over a longer period of time.

The present study tested the following hypotheses:

Hypothesis 1: Participants in the ePREP condition will experience improved mental health outcomes relative to the control/placebo condition.

Hypothesis 2: Participants in the ePREP condition will experience improved relationship outcomes relative to the control/placebo condition.

Hypothesis 3: Gains will be maintained even in the face of relationship dissolution with or without repartnering.

\section{Method}

Participants were 77 introductory psychology students in romantic relationships of 4 months duration or longer who received course credit for their participation. Recruitment announcements indicated that the study examined how relationships change over time. Women made up $71 \%$ of the sample. Ethnic background was distributed as follows: White, 76\%; African-American, 10\%; Hispanic, 7\% and "Other," 7\%. These patterns for gender and ethnicity are consistent with the pool from which the sample was drawn. The average length of relationship for participants was approximately 1 year. Approximately 9\% of the sample reported living together with the remaining 91\% living apart. Participants' ages ranged from 18 to 25 with the average age being 19.4 at the beginning of the study.

At baseline, 8 weeks postbaseline, and approximately 10 months (44 weeks) postbaseline, participants completed questionnaires that assessed symptoms of depression and anxiety (i.e., Beck Depression Inventory, Beck, Steer, \& Garbin, 1988; Beck Anxiety Inventory, Beck, Epstein, Brown, \& Steer, 1988), intimate partner violence (i.e. the nonsevere items from the Negotiation, Psychological Aggression, and Physical Assault subscales from the Revised Conflict Tactics Scale resulting in 38 total items with scale reliabilities ranging from $\alpha=.81$ to $\alpha=.95$, Straus, Hamby, Boney-McCoy, \& Sugarman, 1996), communication patterns, (i.e., Communication Patterns QuestionnaireConstructive Communication Subscale, Heavey, Larson, Zumtobel, \& Christensen, 1996) and relationship satisfaction, (i.e., Couples Satisfaction Index, Funk \& Rogge, 2007). Each of these scales were scored such that higher scores reflect more of the construct of interest.

A computer generated randomization list was used to assign participants to either the ePREP $(n=38)$ or the placebo/control intervention $(n=39)$. After completing their assigned intervention in the researchers laboratory (that took approximately 1 hour), participants in both conditions were given a paper copy of the information covered in the intervention and were informed that they would be contacted by e-mail each week for the next 7 weeks with reminders that if they hoped to gain the maximum benefit from the intervention that they should make an effort to apply the principles learned. Approximately 8 weeks after their initial visit to the lab, participants returned to complete the same battery of questionnaires. Participants were contacted by email again approximately 10 months after baseline and asked to complete an online survey that contained the same battery of questionnaires. A flowchart of the randomized clinical trial design can be seen in Figure 1.

\section{Interventions}

In the relationship focused preventive intervention (ePREP), participants received training in empirically validated methods for improving romantic relationships. ePREP is based on the Prevention and Relationship Enhancement program (PREP, Markman, Stanley, \& Blumberg, 2001), but modified to make it appropriate for a college dating population and computerized administration. An overview of the topics taught in the intervention can be seen in Table 1. The main focus of the intervention is skills training in effective communication techniques and problem-solving skills. It also teaches individuals how to enhance positive aspects of romantic relationships. Participants in the placebo condition viewed a presentation that has served as an effective computer based control/placebo condition in two previous stud-

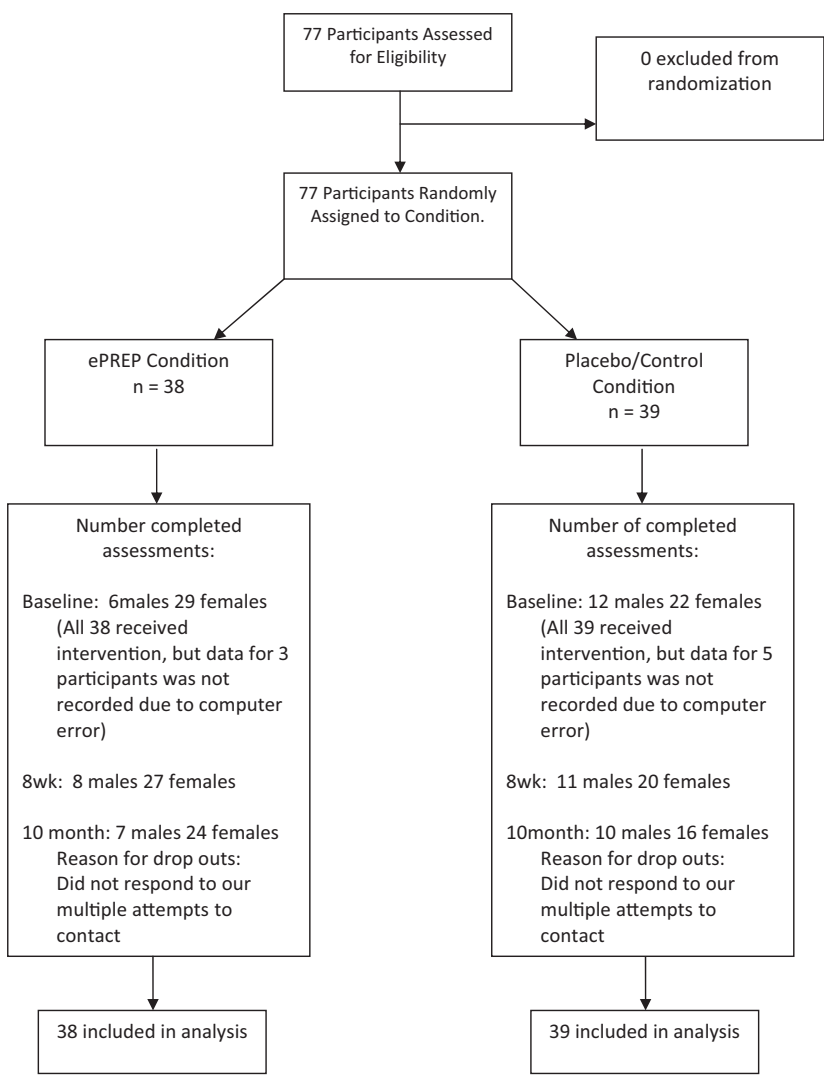

Figure 1. Flowchart of randomized clinical trial design. 
Table 1

Description of Sections of the ePREP Intervention

\begin{tabular}{|c|c|}
\hline Section & Description \\
\hline 1: & $\begin{array}{l}\text { Improving Your Relationship: Explains why it is worth } \\
\text { investing time and effort into improving one's } \\
\text { romantic relationship, introduces static and dynamic } \\
\text { risk factors for relationship distress, communication } \\
\text { dangers signs, and the Time Out Ground Rule } \\
\text { (including when/how to use). }\end{array}$ \\
\hline 2: & $\begin{array}{l}\text { Filters: Explains the concept of filters, how these can } \\
\text { impair good communication and how to effectively } \\
\text { deal with these filters when they arise. }\end{array}$ \\
\hline 3: & $\begin{array}{l}\text { Communication: Teaches XYZ Communication and the } \\
\text { Speaker-Listener Technique. }\end{array}$ \\
\hline 4: & $\begin{array}{l}\text { Issues and Events: Describes the issues and events } \\
\text { model and how to use the ground rules and skills } \\
\text { taught in ePREP to effectively deal with issues and } \\
\text { events as they arise. }\end{array}$ \\
\hline 5: & $\begin{array}{l}\text { Problem Solving: Teaches an effective method for } \\
\text { problem solving that separates problem discussion } \\
\text { (using the Speaker-Listener Technique) and problem } \\
\text { solving (which needs to occur after problem } \\
\text { discussion). }\end{array}$ \\
\hline 6: & $\begin{array}{l}\text { Fun, Friendship and The Foundation of a Good } \\
\text { Relationship: Explains sliding versus deciding, key } \\
\text { considerations in mate selection, gender differences } \\
\text { in patterns of commitment, and a specific method for } \\
\text { enhancing fun and friendship in romantic } \\
\text { relationships. }\end{array}$ \\
\hline 7: & $\begin{array}{l}\text { Ground Rules: Reviews content and describes specific } \\
\text { ways that the skills and ground-rules taught in the } \\
\text { intervention can be optimally employed (e.g. having } \\
\text { regular couple meetings). }\end{array}$ \\
\hline
\end{tabular}

ies (Braithwaite \& Fincham, 2007; Cukrowicz \& Joiner, 2007). Participants in this condition viewed material that provided inert descriptive information about anxiety, depression, and relationship distress such as definitions, prevalence rates, and available forms of treatment.

\section{Results}

\section{Analytic Approach}

The data were analyzed using latent growth curve modeling in Mplus 5. In our model, the loadings from the intercept latent variable to the observed variables were fixed to unity, and each of the paths from the slope latent variable were constrained to be equal to the number of weeks from the baseline assessment $(0,8$, and 44 weeks); this is analogous to centering in hierarchical linear modeling. Condition was included in the model as a fixed covariate, and the variances from the latent variables (intercept and slope) were allowed to covary; thus, providing information about the relationship between initial levels of the dependent variable and the rate of change across time. The latent growth model can be seen in Figure 2. Specifying the model as described provides information about the impact of the ePREP intervention on the patterns of change or "growth" over time. The Mplus software handles missing data using maximum likelihood estimation, thus allowing a growth curve to be fit for all respondents even if they did not have data for each time point (Little \& Rubin, 2002). Table 2 reports the descriptive statistics for the variables examined in the present study. Analyses of those who did not complete assessments at the two follow up points did not reveal any main effects of dropping out on the slopes or intercepts of any of the dependent variables, nor did the pattern of results for any of the dependent variables change when this covariate was included in the model. Results for each of the specified hypotheses are discussed below.

\section{Hypothesis 1}

When the anxiety variables were entered into the model, a negative variance contributed to a nonpositive definite matrix (a Heywood case). Heywood cases are among the most common problems in structural equations modeling, although their causes are not well understood (van Driel, 1978); when they arise, statistical methodologists recommend setting the offending estimate to zero and re-running the analysis (Dillon, Kumar, \& Mulani, 1987). Pursuant to this recommendation, the offending variance was set to zero and the model was re-run. The corrected model provided a good fit to the data that was not significantly different from the uncorrected model $\chi^{2}(d f)=1.275(3), p=.74$, TLI $=$ $1.01, \mathrm{CFI}=1.00, \mathrm{RMSEA}=.00$. In this model, condition predicted slope such that those who received the ePREP intervention had less anxiety over time relative to the placebo/control group $\beta=.369, p<.05$. Slope and intercept were not significantly associated with one another, suggesting that initial levels of anxiety were not associated with the rate of change in anxiety over time. Estimates of the between groups effect sizes on this and each of the examined variables are presented in Table 3.

When depression was entered into the model, the model provided a moderate fit to the data; however, condition did not significantly predict slope, suggesting that the ePREP intervention did not have a detectable effect on depressive symptoms over time. Slope and intercept were not significantly associated for depression scores. Thus, in partial support of the first hypothesis, ePREP produced better outcomes for

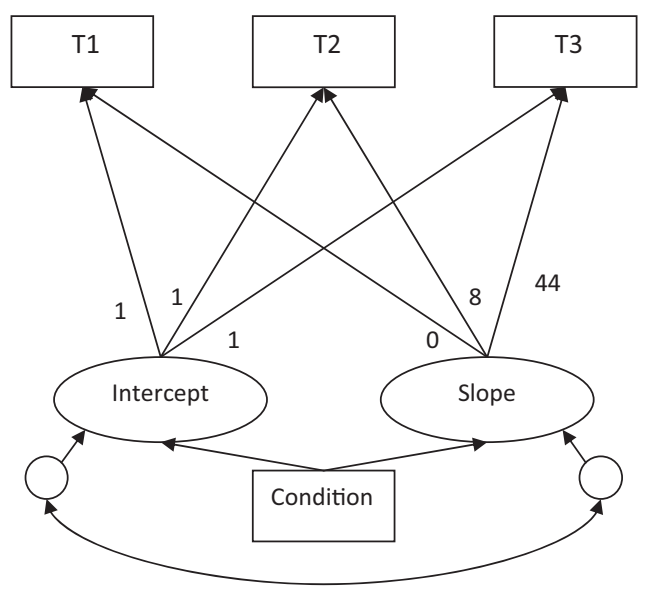

Figure 2. Basic growth curve model. 
Table 2

Estimated Marginal Means and Standard Errors of Measures at Preintervention (Pre), Postintervention (Post) and 10-Month Follow-Up (F/U)

\begin{tabular}{|c|c|c|c|c|c|c|}
\hline \multirow[b]{2}{*}{ Scale } & \multicolumn{3}{|c|}{ ePREP } & \multicolumn{3}{|c|}{ Control } \\
\hline & Pre & Post & $\mathrm{F} / \mathrm{U}$ & Pre & Post & $\mathrm{F} / \mathrm{U}$ \\
\hline FR-CSI & $1.62(.11)$ & $1.87(.16)$ & $1.865(.17)$ & $1.69(.12)$ & $1.71(.18)$ & $1.93(.19)$ \\
\hline BDI & $9.62(1.30)$ & 7.98 (1.53) & $6.79(1.18)$ & $8.88(1.50)$ & $8.06(1.76)$ & 8.44 (1.37) \\
\hline BAI & $9.54(1.43)$ & $11.27(1.91)$ & 5.69 (1.33) & $6.08(1.65)$ & $8.14(2.21)$ & $7.14(1.53)$ \\
\hline CTS-Assault & $.59(.22)$ & $.66(.41)$ & $.08(.40)$ & $.07(.25)$ & $.49(.46)$ & $.68(.45)$ \\
\hline CTS-Psy & $1.09(.31)$ & $1.64(.38)$ & $.83(.36)$ & $.86(.34)$ & $.63(.43)$ & $1.11(.41)$ \\
\hline CTS-Neg & $10.10(1.26)$ & $8.33(1.12)$ & $9.01(1.21)$ & 7.54 (1.37) & $5.97(1.22)$ & $5.55(1.33)$ \\
\hline CPQ & 14.08 (1.97) & $11.96(1.99)$ & $13.21(1.98)$ & $9.76(2.11)$ & $12.86(2.13)$ & $10.76(2.02)$ \\
\hline
\end{tabular}

Note. $\quad$ FR-CSI $=$ Funk-Rogge Couples Satisfaction Index; BDI = Beck Depression Inventory;

BAI $=$ Beck Anxiety Inventory; CTS-Assault $=$ Revised Conflict Tactics Scale Physical Assault

Scale; CTS-Psy = CTS-2 Psychological Aggression Scale; CTS-Neg = CTS-2 Negotiation Scale;

$\mathrm{CPQ}=$ Conflict Patterns Questionnaire-Constructive Communication Scale.

symptoms of anxiety, but the intervention was not significantly associated with changes in depressive symptoms over time.

\section{Hypothesis 2}

When scores for negotiation were entered into the model, a negative variance was generated, thus the offending estimate was set to 0 and the model was re-run. The corrected model provided a good fit to the data that was not significantly different from the uncorrected model $\chi^{2}(d f)=3.603(3), p=.31$, TLI $=.967$, CFI $=.984$, RMSEA $=.051$. Condition did not predict the slope of negotiation scores; however, it did predict the intercept such that those in the placebo condition had lower initial scores $(\beta=-.34, p<.05)$.

When scores for psychological aggression were entered into the model, a negative variance was generated, thus the offending estimate was set to 0 and the model was re-run. The corrected model provided a good fit to the data that was not significantly different from the uncorrected model $\chi^{2}(d f)=4.87(4), p=.30$, TLI $=.974$, CFI $=.983$, RMSEA $=.05$. In this model, condition predicted slope

Table 3

Between Group Effect Sizes at 10-Month Follow-Up (ePREP-Control)

\begin{tabular}{|c|c|c|c|}
\hline Measure & $\begin{array}{l}\text { Bias corrected } \\
\text { effect size } \\
\text { (Hedges'g) }\end{array}$ & $S E$ & $95 \%$ confidence interval \\
\hline FR-CSI & -.36 & .27 & $(-0.88-0.17)$ \\
\hline BDI & -1.28 & .29 & $(-1.85--0.71)$ \\
\hline BAI & -1.00 & .28 & $(-1.56--0.45)$ \\
\hline CTS-Assault & -1.40 & .30 & $(-1.98--0.82)$ \\
\hline CTS-Psy & -.72 & .27 & $(-1.26--0.18)$ \\
\hline CTS-Neg & 2.69 & .37 & $(1.98-3.41)$ \\
\hline $\mathrm{CPQ}$ & 1.21 & .29 & $(0.64-1.78)$ \\
\hline
\end{tabular}

Note. FR-CSI = Funk-Rogge Couples Satisfaction Index; $\mathrm{BDI}=$ Beck Depression Inventory; BAI = Beck Anxiety Inventory; CTS-Assault = Revised Conflict Tactics Scale Physical Assault Scale; CTS-Psy = CTS-2 Psychological Aggression Scale; CTS-Neg = CTS-2 Negotiation Scale; CPQ = Conflict Patterns Questionnaire-Constructive Communication Scale. such that those who received the ePREP intervention evidenced less psychological aggression in their relationship over time relative to the placebo/control group $\mathrm{b}(S E)=$ .015 (.008), $p=.056$ in initial model; b $(S E)=.014, p=$ .074 in corrected model. Unstandardized results from both models are presented because the negative variance in the model was for slope and having a negative variance for slope does not mathematically allow for a meaningful $\beta$ to be generated. Slope and intercept were not significantly associated with one another. To provide an additional view of the rates of psychological aggression in our sample and the clinical significance of the ePREP intervention on psychological aggression, scores were recoded to reflect the presence or absence of any form of psychological aggression (presence $=1$, absence $=0$ ). Table 4 displays the percentages of individuals who reported psychological aggression over time. The percentage of individuals reporting incidences of psychological aggression in the ePREP condition decreased by $12 \%$ over 10 months (from $29 \%$ to $17 \%$ ). The control group decreased by only $3 \%$ (from $25 \%$ to $22 \%$ ).

When scores for physical assault were entered into the model, the data fit the model well $\chi^{2}(d f)=.868(2), p=.65$, $\mathrm{TLI}=.1 .162$, CFI $=1.00$, RMSEA $=.00$; however, a negative variance was generated, thus the offending estimate was set to 0 and the model was re-run. The corrected model provided a poor fit to the data that was significantly different from the uncorrected model $\chi^{2}(d f)=6.398$ (3), $p=.09, \mathrm{TLI}=.675, \mathrm{CFI}=.838, \mathrm{RMSEA}=.121 ; \chi^{2 \text { Diff }}$

Table 4

Between Group Comparison of Percentages of Any Form of Physical Assault and Psychological Aggression

\begin{tabular}{lccccccc}
\hline \multirow{2}{*}{ Scale } & \multicolumn{3}{c}{ ePREP } & & \multicolumn{3}{c}{ Control } \\
\cline { 2 - 4 } \cline { 6 - 8 } & Pre & Post & F/U & & Pre & Post & F/U \\
\hline CTS-Assault & $43 \%$ & $37 \%$ & $17 \%$ & & $21 \%$ & $29 \%$ & $15 \%$ \\
CTS-Psy & $29 \%$ & $26 \%$ & $17 \%$ & & $25 \%$ & $22 \%$ & $22 \%$ \\
\hline
\end{tabular}

Note. $\quad$ CTS-Assault $=$ Revised Conflict Tactics Scale Physical Assault Scale; CTS-Psy = CTS-2 Psychological Aggression Scale. 
$(d f)=5.53(1), p<.05$. As such, the results for the test of Hypothesis 2 are presented for both models. For the uncorrected model, a trajectory of less physical assault emerged for those in the ePREP condition, $\beta=.169, p=.051$. The results for the corrected model were similar with ePREP predicting a trajectory of less physical assault $\beta=.284, p=$ .055. Condition also predicted intercept such that individuals in the placebo/control condition had lower initial physical assault scores in the uncorrected model $\beta=-.354, p=$ .053 ; and the corrected model $\beta=-.411, p=.053$. There was a nonsignificant relationship between slope and intercept in both models. To provide an additional view of the rates of physical assault in our sample and the clinical significance of the ePREP intervention on physical assault, scores were recoded to reflect the presence or absence of any form of physical assault (presence $=1$, absence $=0$ ). Table 4 displays the percentages of individuals who reported physical assault over time. This transformation revealed that the percentage of individuals reporting incidences of physical assault in the ePREP condition decreased by $26 \%$ over 10 months (from $43 \%$ to $17 \%$ ). The control group decreased by only $6 \%$ (from $21 \%$ to $15 \%$ ).

For constructive communication and relationship satisfaction, the data provided a good fit to the models; however, condition did not predict the slope for either variable, suggesting that the ePREP intervention did not significantly influence the rate of change on these variables over time. There was, however, a significant association between intercept and slope for relationship satisfaction such that those who had lower initial relationship satisfaction had sharper declines in relationship satisfaction over time $\mathrm{r}=$ $-.42, p<.01$.

Analyses were also run to test for gender effects on each of the dependent variables. Analysis with a dummy variable for gender $($ male $=1$, female $=2$ ) showed that gender was significantly related to the intercept of relationship satisfaction such that the women in our sample had significantly lower initial levels of relationship satisfaction $(\beta=-.716, p<.05)$. Gender was not significantly associated with the slope or intercept of any other variable tested in the present study. Including gender in the model did not change the pattern of results for the associations between condition and slope for any of the dependent variables.

\section{Hypothesis 3}

Breaking up a problematic relationship before getting married and/or having children is a healthy and normative process in mate selection, thus we were not interested in whether ePREP reduced relationship dissolutions per se. However, it is important to know whether or not the gains achieved through ePREP are dependent on the specific relationship that was improved by the intervention or whether these gains are durable in the face of relationship dissolution. To test this, analyses were re-run with a dummy variable included in the model that indicated whether the participant was in the same relationship as they were at the beginning of the study (same $=1$ ) or not $($ not same $=0)$. At 8 weeks postbaseline, $68 \%$ of participants in the ePREP condition reported that they were in the same relationship as when they began the study compared to $62 \%$ of participants in the placebo/ control condition. At the 10-month follow up, 50\% of participants in the ePREP condition reported that they were in the same relationship as when they began the study compared to $38 \%$ in the placebo control condition. $t$ tests indicated that the rate of relationship dissolution between groups was not statistically significant for either time point. Individuals who ended their relationship were more likely to have lower relationship satisfaction intercepts at baseline $\beta=-.37, p<.01$. Similarly, this covariate predicted the slopes for constructive communication $(\beta=.98, p=.06)$ and negotiation $(\beta=.65, p<$ $.05)$ such that those who stayed in the same relationship evidenced more constructive communication and negotiation over time regardless of condition. Including the "break up" covariate in the model did not significantly attenuate any of the significant relationships between condition and slope, suggesting that the positive impact of ePREP is durable to relationship dissolution.

To rule out the possibility that being in a relationship (whether it was with the same person or not) could be driving the positive mental health findings, we created another dummy variable that indicated whether or not the participants were in a current relationship (current relationship $=1$ ) or not (no relationship $=0$ ) at the final assessment. This is an important variable to consider because research has shown that being in committed dating relationships provides health and psychological benefits that nondaters do not experience (Braithwaite, Delevi, \& Fincham, in press). Including this covariate in the model did not significantly attenuate any of the significant relationships between relationship and slope for the mental health variables, nor did this covariate significantly predict slopes or intercepts for any of the dependent variables. These findings suggest that the impact of the ePREP intervention on mental health outcomes is durable to relationship dissolution without repartnering.

\section{Discussion}

The present study replicated the efficacy of the ePREP intervention for mental health and relationship relevant outcomes. It extended previous research by showing, at a 10-month follow up, that participants in the ePREP condition experienced improved mental health and relationship relevant outcomes for many although not all, variables examined in the present study. It further extends prior findings by showing that the impact of the ePREP intervention on these outcomes was durable to relationship dissolution whether those that ended their relationships repartnered or not.

An interesting pattern emerged in the results such that individuals in the ePREP condition seemed to get worse before they got better. This pattern was observed in the anxiety, physical assault, psychological aggression, and constructive communication variables. It is possible that 
this pattern of results emerged because of the implementation of new skills to the participants' relationships; specifically, that using new communication skills unearthed issues that had not been previously addressed. It is interesting that this pattern seemed to occur in variables where the intervention had the most success at changing outcomes. Perhaps the active ingredients of the ePREP intervention have an incubation period such that they create initial increases in problematic outcomes but ultimate improvement in outcomes over longer spans of time. This could also be a consequence of the individual, rather than the couple focus, of the intervention; perhaps because only one member of the dyad is receiving the intervention, the skills take longer to be advantageously employed between both members of the dyad. Future research using couples could further illuminate this issue.

Notwithstanding its positive findings, the present study is limited by the self-report nature of our data, and a lack of optimal power because of a small sample in which women were overrepresented. On the other hand, the sample was ethnically diverse, representative of the college population from which it was drawn, and college students have been targeted by relationship researchers as a key group for relationship education (Ooms \& Wilson, 2004). Another point worth noting is that our study is not a traditional primary prevention study wherein well individuals are tracked over time and between-group differences of welldefined, dichotomous criteria (e.g. diagnoses of major depressive disorder or divorce) are compared; rather, we took well individuals and showed that their outcomes (measured on continuous scales) across 10 months were superior to individuals who received a placebo control. Using this methodology, we found that our intervention significantly reduced incidence of physical assault and psychological aggression, among other variables; thus, these findings are important in their own right. However, future research may profitably examine the impact of ePREP using methodologies that more closely approximate traditional primary prevention studies.

Although research is needed to determine what kind of an impact ePREP may have when administered to couples, it is noteworthy that an hour long, self-directed intervention completed by one partner produced changes in mental health and relationship relevant variables at a 10-month follow up. This is encouraging when one considers the important implications for dissemination. Often, both members of a relationship dyad are not willing to participate in relationship interventions. Because ePREP has been shown to be efficacious when delivered to only one member of the dyad, it has the power to overcome this obstacle. Further, its efficacy for individuals makes it possible to administer the intervention to large swaths of target populations (such as college students) since it does not require that both members of the dyad participate to produce results.

Finally, the results of this study have implications for public health interventions. ePREP is the only flexible form of relationship education that has replicated its efficacy for improving both relationship and mental health outcomes over longer spans of time. ePREP addresses the two problems facing relationship education; specifically, its flexibil- ity allows easier access to target populations (even when both members of the dyad do not participate), and ePREP is ideally suited to help maintain gains from traditional relationship education when used as an adjunct "booster" treatment. Thus, policy makers who are interested in cost and time-effective ways to improve outcomes across multiple domains may be particularly interested in the flexibility and cross-domain impact offered by ePREP.

\section{References}

Beck, A. T., Epstein, N., Brown, G., \& Steer, R. A. (1988). An inventory for measuring clinical anxiety: Psychometric properties. Journal of Consulting and Clinical Psychology, 56, 893-897.

Beck, A. T., Steer, R. A., \& Garbin, M. G. (1988). Psychometric properties of the Beck Depression Inventory: Twenty-five years of evaluation. Clinical Psychology Review, 8, 77-100.

Braithwaite, S. R., Delevi, R. D., \& Fincham, F. D. (in press). Romantic relationships and the physical and mental health of college students. Personal Relationships.

Braithwaite, S. R., \& Fincham, F. D. (2007). ePREP: Computer based prevention of relationship dysfunction, depression and anxiety. Journal of Social and Clinical Psychology, 26, 609-622.

Cavanagh, K., \& Shapiro, D. A. (2004). Computer treatment for common mental health problems. Journal of Clinical Psychology, 60, 239-251.

Cukrowicz, K. C., \& Joiner, T. E. (2007). Computer-based intervention for anxious and depressive symptoms in a non-clinical population. Cognitive Therapy and Research, 31, 677-693.

Dillon, W. R., Kumar, A., \& Mulani, N. (1987). Offending estimates in covariance structure analysis: Comments on the causes of and solutions to Heywood cases. Psychological Bulletin, 101, $126-135$.

Funk, J. L., \& Rogge, R. D. (2007). Testing the ruler with item response theory: Increasing precision of measurement for relationship satisfaction with the couples satisfaction index. Journal of Family Psychology, 21, 572-583.

Halford, W. K., Moore, E., Wilson, K. L., Dyer, C., \& Farrugia, C. (2004). Benefits of a flexible delivery relationship education: An evaluation of the couple CARE program. Family Relations, 53, 469.

Heavey, C. L., Larson, B. M., Zumtobel, D. C., \& Christensen, A. (1996). The communication patterns questionnaire: The reliability and validity of a constructive communication subscale. Journal of Marriage and the Family, 58, 796-800.

Jakubowski, S. F., Milne, E. P., Brunner, H., \& Miller, R. B. (2004). A review of empirically supported marital enrichment programs. Family Relations, 53, 528-536.

Larson, J. H., Galbraith, R. C., Holman, T. B., \& Stahmann, R. F. (2007). The RELATionship evaluation (RELATE) with therapistassisted interpretation: Short-term effects on premarital relationships. Journal of Marital and Family Therapy, 33, 364-374.

Little, R. J., \& Rubin, D. B. (2002). Statistical analysis with missing data (2nd edition). New York: John Wiley \& Sons.

Markman, H. J., Renick, M. J., Floyd, F. J., \& Stanley, S. M. (1993). Preventing marital distress through communication and conflict management training: A 4- and 5-year follow-up. Journal of Consulting and Clinical Psychology, 61, 70-77.

Markman, H. J., Stanley, S. M., \& Blumberg, S. L. (2001). Fighting for your marriage. San Francisco: Jossey-Bass.

Ooms, T., \& Wilson, P. (2004). The challenges of offering relationship and marriage education to low-income populations. Family Relations, 53, 440-447.

Straus, M. A., Hamby, S. L., Boney-McCoy, S., \& Sugarman, 
D. B. (1996). The Revised Conflict Tactics Scales (CTS2): Development and preliminary psychometric data. Journal of Family Issues, 17, 283-316.

Sullivan, K. T., \& Bradbury, T. N. (1997). Are premarital prevention programs reaching couples at risk for marital dysfunction? Journal of Consulting and Clinical Psychology, 65, $24-30$ van Driel, O. P. (1978). On various causes of improper solutions in maximum likelihood factor analysis. Psychometrika, 43, 225243.

Received January 22, 2008

Revision received July 14, 2008

Accepted July 28, 2008

\section{American Psychological Association} SUBSCRIPTION Claims INFORMATION

Today's Date:

We provide this form to assist members, institutions, and nonmember individuals with any subscription problems. With the appropriate information we can begin a resolution. If you use the services of an agent, please do NOT duplicate claims through them and directly to us. PLEASE PRINT CLEARLY AND IN INK IF POSSIBLE.

PRINT FULL NAME OR KEY NAME OF INSTITUTION

$\overline{\text { ADDRESS }}$

CITY

YOUR NAME AND PHONE NUMBER

TITLE
MEMBER OR CUSTOMER NUMBER (MAY BEFOUND ON ANY PAST ISSUE LABEL)

DATE YOUR ORDER WAS MAILED (OR PHONED)

PREPATD CHECK CHARGE

CHECK/CARD CLEARED DATE:

(If possible, send a copy, front and back, of your cancelled check to help us in our research of your clainn.)

ISSUES: MISSDNG DAMAGED

VOLUME OR YEAR NUMBER OR MONTH

Thank you. Once a claim is received and resolved, delivery of replacement issues routinely takes 4-6 weeks.

(TO BE FILLED OUT BY APA STAFF)

DATE RECEIVED:

ACTION TAKEN:

STAFF NAME:
DATE OF ACTION:

INV. NO. \& DATE:

LABEL NO. \& DATE:

Send this form to APA Subscription Claims, 750 First Street, NE, Washington, DC 20002-4242

PLEASE DO NOT REMOVE. A PHOTOCOPY MAY BE USED. 